\title{
The establishment of human anti-TROP2 antibody IgG and its inhibition function on pancreatic cancer cell proliferation
}

\author{
Huan Wang ${ }^{1}$, Xiaojun Tang ${ }^{2}$, Xin $\mathrm{Xu}^{3}$, Chu $\mathrm{Chu}^{4}{ }^{4}$, Siping Xiong ${ }^{2}$, Feng Zheng ${ }^{5}$, Qisong Peng ${ }^{1 *}$ \\ ${ }^{1}$ Department of Laboratory Medicine, The Affiliated Jiangning Hospital of Nanjing Medical University, Nanjing, Jiangsu, 211100, China; \\ ${ }^{2}$ Key Laboratory of Antibody Technique of Ministry of Health, Nanjing Medical University, Nanjing, Jiangsu, 211166, China; \\ ${ }^{3}$ School of Medical Science and Laboratory Medicine, Jiangsu University, Zhenjiang, Jiangsu, 212001, China; \\ ${ }^{4}$ Nanjing Maternity and Child Health Hospital, Nanjing, Jiangsu, 210004, China; \\ ${ }^{5}$ Huadong Medical Institute of Biotechniques, Nanjing, Jiangsu, 210002, China.
}

\begin{abstract}
On the basis of anti-TROP2 Fab antibody, this study seek to construct a eukaryotic expression system of human anti-TROP2 antibody IgG, and to analyze the inhibition function of human anti-TROP2 antibody IgG in the cell proliferation of pancreatic cancer. The heavy and light chain genes of anti-TROP2 antibody were amplified respectively to establish the recombinant expression vector of human anti-TROP2 antibody IgG, named pWSanti-TROP2. The human anti-TROP2 antibody IgG was obtained through transfecting the plasmids into the CHO $\mathrm{dhfr}^{-}$cell line, selecting the monoclonal cell strains with high amounts of antibody expression by MTX screening and applying Protein $G$ affinity in purification. The identification and immunologic activity of human antiTROP2 antibody IgG were researched by Western blot, SDS-PAGE, ELISA, immunofluorescence assay and flow cytometry method (FCM). MTT assay was conducted to analyze the inhibition effect of human anti-TROP2 antibody IgG on BxPC3 cell proliferation. The human anti-TROP2 antibody IgG eukaryotic expression system was established successfully to express human anti-TROP2 antibody IgG, in which the molecular weight of heavy chain and light chain were consistent with expectation, and it could specifically combine with TROP2 protein, the antibody titer reaching 1:6,400. The MTT assay results indicated that human anti-TROP2 antibody IgG had a significant effect on inhibiting the proliferation of BxPC3 cell, and the inhibition function can be gradually increased with improved antibody dose and prolonged time. In the study, the human anti-TROP2 antibody IgG eukaryotic expression system was constructed successfully, the antibody could specifically bind to TROP2 protein on the surface of pancreatic cancer cells, and it is shown to have a significant inhibitory action in pancreatic cancer cell proliferation.
\end{abstract}

Keywords: TROP2, CHO $\mathrm{dhfr}^{-}$cell line, eukaryotic expression system, cell proliferation, pancreatic cancer

\section{INTRODUCTION}

Pancreatic cancer is both a highly malignant and a common tumor, its pathogenesis involves the mu-

\footnotetext{
$\triangle$ Correspondence to: Qisong Peng, Department of Laboratory Medicine, The Affiliated Jiangning Hospital of Nanjing Medical University, 168 Gushan Road, Jiangning, Nanjing 211100, China. Tel:86-2552281848-81040. Email:pengqs520@163.com.

The authors reported no conflict of interest.
}

tation and abnormal expression of many genes ${ }^{[1-3]}$. Some research reported that the high expression of trophoblast cell-surface antigen 2 (TROP2) is related to the occurrence and development of pancreatic cancer. TROP2 gene is located in $1 \mathrm{p} 32$ area without intron gene, and encode the protein which contains 323 amino acids and molecular weight is $35.7 \mathrm{kDa}^{[4,5]}$. TROP2 is a single transmembrane glycoprotein, which is rarely expressed or absent in normal epithelial tissues, while highly expressed in trophoblast cells 
on pancreatic cancer cell proliferation, 2017, 1(4)

and epithelial carcinoma. The research shows that TROP2 is closely correlated with the invasion of many kinds of tumors, and is able to promote the invasion and metastasis of tumor cells ${ }^{[6]}$. Therefore, TROP2 may become a new marker for tumor prognosis, and a potential target in tumor targeted therapy ${ }^{[7,8]}$. In this light, the study of anti TROP2 antibody has very important clinical significance. While the anti-TROP2 antibody obtained from prokaryotic expression system has many disadvantages, e.g. the biological activity of its expression product is relatively low, the antibody fragments are likely to produce toxic effects on the host cells, and so on. In addition, studies found that small molecule antibodies (such as the Fab antibody) have many shortcomings including short retention time, easy cleaning, and so on. Therefore based on the anti-TROP2 Fab antibody made in our laboratory, this study established a human anti-TROP2 antibody IgG recombinant expression vector, transfected the plasmid into $\mathrm{CHO} \mathrm{dhfr}^{-}$cells, expressed a human anti-TROP2 antibody IgG, and observed the specific binding activity with TROP2 protein on the pancreatic cancer cell surface and the inhibitory effect on the proliferation of pancreatic cancer cells, hopefully laying the foundation for the targeted therapy of pancreatic cancer ${ }^{[9-12]}$.

\section{MATERIALS AND METHODS}

\section{Materials}

Chinese hamster ovary cell lines $\mathrm{CHO} \mathrm{dhfr}^{-}$, pancreatic cancer cell line BxPC 3 and the eukaryotic expression system of pWS-2 were maintained in the Key Laboratory of Antibody Technique of the Ministry of Health, Nanjing Medical University, Nanjing, China. Ham's F12 nutrient medium (F12), Dulbecco's modified Eagle's medium (DMEM) and fetal bovine serum (FBS) were supplied by Gibco (USA). E.coli.
XL1-Blue, $0.25 \%$ trypsin EDTA, $100 \times$ penicillin/ streptomycin (double-antibody) were purchased from Invitrogen (USA). Rabbit-anti-human IgG was purchased from Boster Biological Technology Co., Ltd., (China), HRP labeled anti-human Fab, HT and MTX were purchased from Sigma (Japan), recombinant human TROP2 protein was purchased from R\&D (USA), and the restriction enzymes Nhe I , Mlu I , Not I ,EcoR I and T4 DNA ligase were supplied by NEB (USA), TMB was supplied by Pierce (USA), DNA Marker was obtained from TaKaRa (Japan), DNA transfection reagent was supplied by Roche(Switzerland), and $0.22 \mu \mathrm{m}$ filter (30 k) were purchased from Millipore (USA), Protein G (5 $\mathrm{mL}$ ) affinity column was obtained from GE(USA), and plasmid purification kit and DNA gel extraction kit were purchased from QIAGEN (Germany), cell culture bottle and 96 microtitor plate were purchased from Corning (USA).

\section{Establishment of the eukaryotic expression system of human anti-TROP2 antibody IgG}

The light chain gene fragment was amplified by using the laboratory constructed anti-TROP2 Fab antibody gene as the template. Fd gene fragment was amplified by using the anti-TROP2 Fab gene as above, and the stable region gene fragment of $\mathrm{CH} 2-\mathrm{CH} 3$ was amplified from the $\mathrm{pT}-\mathrm{CH} 23$ plasmid. The restriction enzymes EcoR I and Not I were used in Fd gene fragment and $\mathrm{CH} 2-\mathrm{CH} 3$ gene fragment, then they were linked by $\mathrm{T} 4$ ligase and the plasmid was added to the competent cells of E.coli. XL1-Blue. Positive colonies were selected to undergo PCR to obtain the right recombinant vector plasmid. The heavy chain gene was amplified from the plasmid with right gene sequences of $\mathrm{Fd}$ and $\mathrm{CH} 2-\mathrm{CH} 3$ gene fragments by using overlap PCR. All primer sequences were shown in Table 1.

Table 1 The sequences of primers used in PCR

\begin{tabular}{|c|c|}
\hline Target genes & Primers \\
\hline Light chain gene & $\begin{array}{l}\text { Forward: 5'-GCTAGCGCCGCCACCATGGACATGCGGGTTCCAGCCCAGCTTCTCGGACTTCTGCTGTT- } \\
\text { GTGGCTGCGCGGAGCACGGTGCGAGCTCCAGATGACCCAG-3' } \\
\text { Reverse: 5'-ACGCGTCTAACACTCTCCCCTGTTGAAGCTC-3' }\end{array}$ \\
\hline Fd gene fragment & $\begin{array}{l}\text { Forward: 5'-GAATTCGCCGCCACCATGGAGTTCGGACTCAGTTGGCTGTTCCTGGTGGCCATCCTGAAG } \\
\text { GGTGTGCAGTGTCAGGTGCAGCTGGTGGAG-3' } \\
\text { Reverse: 5'-GGGCACGGTGGGCATGTGTGAGTTTTGTCACAAGATTTGGGC-3' }\end{array}$ \\
\hline $\mathrm{CH} 2-\mathrm{CH} 3$ gene fragment & $\begin{array}{l}\text { Forward: 5'-GCCCAAATCTTGTGACAAAACTCACACATGCCCACCGTGCCC-3' } \\
\text { Reverse: 5'-GCGGCCGCTCATTTACCCGGAGACAGGG-3' }\end{array}$ \\
\hline $\begin{array}{l}\text { Heavy chain gene amplified } \\
\text { by overlap PCR }\end{array}$ & $\begin{array}{l}\text { Forward: 5'-GAATTCGCCGCCACCATGGAGTTCGGACTCAGTTGGCTGTTCCTGGTGGCCATCCTGAA- } \\
\text { GGGTGTGCAGTGTCAGGTGCAGCTGGTGGAG-3' } \\
\text { Reverse: 5'-GCGGCCGCTCATTTACCCGGAGACAGGG-3' }\end{array}$ \\
\hline
\end{tabular}


The light chain gene fragment was cloned into the expression vector pWS-2, which introduced the restriction enzymes Nhe I and $M l u \mathrm{I}$, they were linked by T4 ligase and the plasmid was added to the competent cells of E.coli.XL1-Blue. Positive colonies were selected to undergo PCR to obtain the right recombinant vector, known as pWS-L. The heavy chain gene fragment was cloned into the recombinant vector pWS-L, which introduced the restriction enzymes Not I and EcoR I. They were linked by $\mathrm{T} 4$ ligase and the plasmid was added to the competent cells of E.coli.XL1-Blue. The positive colonies were again selected to undergo PCR to obtain the right recombinant vector, which was the recombinant expression vector of human anti-TROP2 antibody IgG, labled as pWS-anti-TROP2.

\section{Expression and purification of human anti- TROP2 antibody IgG}

Optimization of transfection conditions for eukaryotic cells

pEGFP-C3 plasmid was transfected into $\mathrm{CHO} \mathrm{dhfr}^{-}$ cells with different ratios of $\mathrm{pEGFP}-\mathrm{C} 3$ plasmid $(\mu \mathrm{g})$ and Lipofectamine $2000(\mu \mathrm{L})$, the fluorescence intensity was observed under the microscope to select the suitable ratio when fluorescence intensity was the strongest.

\section{Establishment and selection of the monoclonal cell lines with the highest expression}

The pWS-anti-TROP2 plasmid was transfected into $\mathrm{CHO} \mathrm{dhfr}^{-}$cells in equimolar amounts in 6-well plates using Lipofectamine 2000 reagent in suitable ratio. Methotrexate (MTX) was added after $24 \mathrm{~h}$ with ranged concentration for selecting the positive cell lines. Surviving cells from the MTX treatment were plated in 96-well plates for selecting the monoclonal cell strains. After two weeks, the positive cell lines were harvested and cultured, supernatants were obtained to detect the expression of human anti-TROP2 antibody IgG, and the monoclonal strains with higher expression were obtained.

\section{Purification of human anti-TROP2 antibody IgG}

A large amount of these higher expressed monoclonal strains were cultured. When the cell density reached $70 \% \sim 80 \%$ in the culture bottle, serum free medium was applied to the culturing cells until all cells died. Then supernatants were collected by centrifugation, $0.22 \mu \mathrm{m}$ needle type filter was used to filter out impurities and bacteria, and Protein G HP affinity chromatography column was applied to $\mathrm{pu}-$ rifying supernatants to obtain purified human antiTROP2 antibody IgG.
Identification and the immunological function of human anti-TROP2 antibody IgG Identification of human anti-TROP2 antibody IgG

SDS-PAGE and Western blot assay were conducted to analyze the molecular weight of human anti-TROP2 antibody IgG. Purified antibody was used as antigen, while HRP labeled anti-human Fab was used as antibody. Enzyme-linked immuno sorbent assay(ELISA) was used to analyze the antibody titer of human anti-TROP2 antibody IgG. The recombinant human TROP2 protein was coated in ELISA plate after the sealing of $5 \%$ milk powder and rabbit anti human IgG. The purified antibody with a ranged concentration was used as the first antibody, and supernatant from $\mathrm{CHO} \mathrm{dhfr}^{-}$cells without transfection was used as the negative control, then HRP labeled anti-human Fab was added as the second antibody.

\section{Immunological function of human anti- TROP2 antibody IgG}

The immunological function of human anti-TROP2 antibody $\operatorname{IgG}$ was analyzed by flow cytometry and immunofluorescence with BxPC3 and NIH3T3 cell lines. The purified antibody was used as the first antibody, then FITC labeled goat-anti-human IgG was added as the second antibody, and in immunofluorescence, rhodamine labeled anti-human Fab was added as the second antibody.

Inhibition effect of human anti-TROP2 antibody IgG on pancreatic cancer cell proliferation

The inhibition effect of human anti-TROP2 antibody IgG in pancreatic cancer cell was analyzed by cell proliferation assay with BxPC3 and NIH3T3 cell lines. BxPC3 cells and NIH3T3 cells were seeded at $1 \times 10^{4}$ cells/well in $96-$ well plates (four replica wells per data point), purified antibody with ranged concentration was added as the first antibody, rabbitanti-human IgG were taken as the negative control, after $48 \mathrm{~h}, 20 \mu \mathrm{L}$ MTT $(5 \mathrm{mg} / \mathrm{mL})$ was added, after $4 \mathrm{~h}$, supernatant was removed and $200 \mu \mathrm{L} 10 \%$ DMSO was added, the inhibition rate was obtained by multimode reader with the detection wavelength of $490 \mathrm{~nm}$. The inhibition rate $=(1-\mathrm{OD}$ value of the experimental group / OD value of the control group) $\times 100 \%$.

\section{Statistical analysis}

Quantitative results were shown as mean \pm SD. Single-factor variance analysis was adopted for the inhibitory effect of human anti-TROP2 antibody IgG in different cells, and linear correlation analysis was 
used to analyze the correlation between the antibody concentration and the growth inhibition of target cell. $P<0.05$ was considered significant.

\section{RESULTS}

\section{Construction of the eukaryotic expression system of human anti-TROP2 antibody IgG}

The light chain gene fragment was amplified with the specific primers. Through SDS-PAGE, the molecular weight of the products were verified to be about 750 bp (Fig. 1A, $\boldsymbol{a})$, and the gene sequences were verified to be correct. The Fd gene fragment and $\mathrm{CH} 2-\mathrm{CH} 3$ gene fragment were amplified with the specific primers. Through SDS-PAGE, the molecular weight of the products were verified to be about 650 bp (Fig. 1A, b). The products after the double diges- tion (EcoR I and Not I ) were transformed into E.coli. XL1-Blue-competent cells. Finally, we obtained plasmids containing $\mathrm{Fd}$ gene fragment and $\mathrm{CH} 2-\mathrm{CH} 3$ gene fragment. The gene sequences were verified as correct. The heavy chain gene was amplified by overlap PCR and the molecular weight of the products were verified to be about 1,300 bp (Fig. 1A, c).

The light chain gene was cloned into the pWS2 vector, which introduced the restriction enzymes Nhe I and Mlu I , and the recombinant vector was transformed into the competent cells of E.coli.XLlBlue. Finally, the plasmids containing TROP2 gene, labeled pWS-L plasmid, were obtained, and the gene sequences were verified to be correct. Then the heavy chain gene was cloned into the pWS-L vector, which introduced the restriction enzymes Not I and EcoR I , and the recombinant vector was trans-
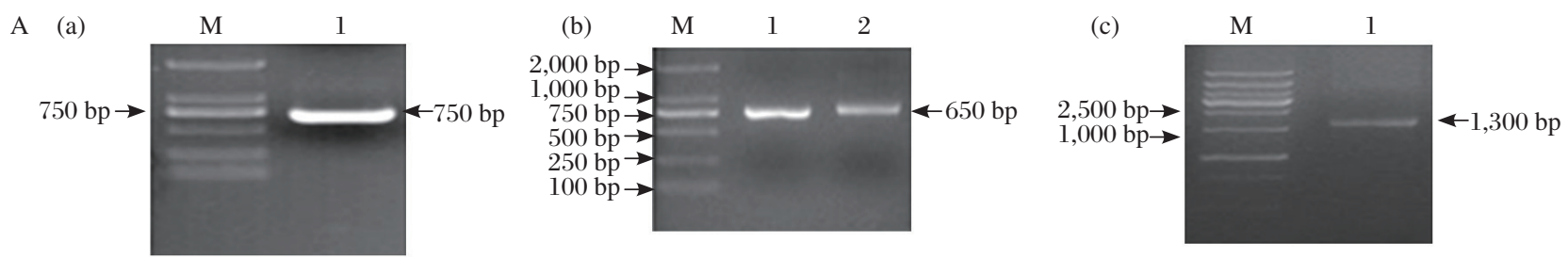

B
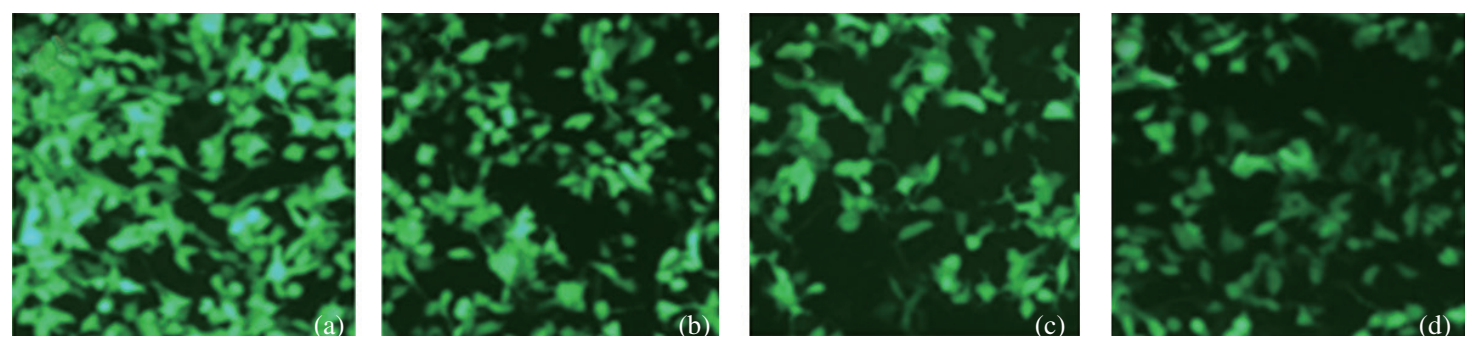

Fig. 1 Construction of the eukaryotic expression system of human anti-TROP2 antibody IgG. A: The PCR products of the establishment of the eukaryotic expression system of human anti-TROP2 antibody IgG. (a) the light chain gene fragment was amplified by PCR, M: DL 2000 Marker,1: the light chain gene fragment; (b) the Fd and CH2-CH3 gene fragment were amplified by PCR, M: DL 2000 Marker, 1: CH2-CH3 gene fragment, 2: Fd gene fragment; (c) the heavy chain gene fragment was amplified by PCR, M: DL 15000 Marker, 1: the heavy chain gene fragment. B: Optimization of cell transfection conditions. (a)-(d): the ratios of pEGFP-C3 plasmid ( $\mu$ g) and Lipofectamine 2000 ( $\mu$ L) were 1:1, 1:2, 1:3 and 1:4.

formed into the competent cells of E.coli.XL1-Blue. Finally, we obtained plasmids containing TROP2 gene and heavy chain gene, labeled pWS-anti-TROP2 plasmid, and the gene sequences were verified correct.

\section{Expression and purification of human anti- TROP2 antibody IgG}

The optimization of transfection conditions for eukaryotic cells

pEGFP-C3 plasmid was transfected into $\mathrm{CHO}$ $\mathrm{dhfr}^{-}$cells with different ratios of pEGFP-C3 $\operatorname{plasmid}(\mu \mathrm{g})$ and Lipofectamine $2000(\mu \mathrm{L})$, fluorescence intensity was observed under the microscope. The results showed that the highest transfection efficiency was obtained when the volume ratio of
pEGFP-C3( $\mu \mathrm{g})$ plasmid and Lipofectamine 2000( $\mu \mathrm{L})$ was 1:1 (Fig.1B).

\section{The establishment and selection of the mono- clonal cell lines with the highest expression}

pWS-anti-TROP2 plasmid was transfected into CHO dhfr ${ }^{-}$cells and MTX in the selected concentration was added after $24 \mathrm{~h}$ for selecting the positive cell lines. After two weeks, supernatant of the positive cell lines was obtained to detect the expression of human anti-TROP2 antibody $\operatorname{IgG}$, and the monoclonal strains with higher expression were harvested.

Purification of human anti-TROP2 antibody IgG

A large amount of supernatant were obtained, purified human anti-TROP2 antibody IgG was collected 
though Protein $\mathrm{G}$ affinity column $(5 \mathrm{~mL})$, and the protein concentration was $4 \mathrm{mg} / \mathrm{mL}$.

\section{Identification and the immunological func- tion of human anti-TROP2 antibody IgG}

Identification of human anti-TROP2 antibody IgG

Human anti-TROP2 antibody IgG was analyzed by SDS-PAGE and Western blot. The results showed target strips at $55 \mathrm{kDa}$ and $28 \mathrm{kDa}(\boldsymbol{F i g}$. $2 \boldsymbol{A}$ ). The antibody titer detection results demonstrated that human anti-TROP2 antibody IgG could specifically combine with TROP2 antigen in 1:6,400 dilution (Fig. $2 \boldsymbol{B}$ ).

\section{Immunological function of human anti- TROP2 antibody IgG}

The results of flow cytometry showed that the binding rate of NIH3T3 cells and human anti-TROP2 antibody $\operatorname{IgG}$ was $2.27 \%$, and the binding rate of BxPC3 cells and human anti-TROP2 antibody IgG was $85.99 \%$ (Fig. 2C).

The results of immunofluorescence detection demonstrated that human anti-TROP2 antibody IgG was located on the cell membrane of BxPC3 cells (green fluorescence), while there was no green fluorescence on NIH3T3 cells. DAPI was located on the nucleus (blue fluorescence) (Fig. 2D).

Inhibition effect of human anti-TROP2 antibody IgG on pancreatic cancer cell proliferation

MTT results showed that when the dilution of human anti-TROP2 antibody IgG were 1:5, 1:10, 1:20,
$1: 40$ and $1: 80$, the inhibition rates of the antibody to BxPC3 cells were $75.81 \% \pm 6.76 \%, 50.38 \% \pm 3.95 \%$, $41.41 \% \pm 2.29 \%, 27.35 \% \pm 3.36 \%$ and $15.29 \% \pm 2.64 \%$ respectively, while the inhibition rates of the antibody to NIH3T3 cells were $6.14 \% \pm 1.47 \%, 5.97 \% \pm 1.35 \%$, $3.14 \% \pm 0.98 \%, 2.75 \% \pm 1.15 \%$ and $5.50 \% \pm 1.98 \%$ (Fig. 3A). The inhibition rates of human anti-TROP2 antibody $\mathrm{IgG}$ to BxPC3 cells and NIH3T3 cells were analyzed by one-way ANOVA, the results showed that the inhibitory effect on BxPC3 cells decreased with the increased dilution, and there was basically no inhibitory effect on NIH3T3 cells, which indicated that human anti-TROP2 antibody IgG had an obvious inhibitory effect on the growth and proliferation of BxPC3 cells, however, it basically had no inhibitory effect on NIH3T3 cells, the differences were statistically significant $(P<0.05)$.

The results of the correlation analysis of antibody concentration and inhibition rates demonstrated that $R^{2}$ was equal to 0.9691 on BxPC3 cells, and $R^{2}$ was equal to 0.1921 on NIH3T3 cells (Fig. $3 \boldsymbol{B}$ ), which indicated that there was a dose dependent relationship between antibody concentration and inhibition rates of BxPC3 cells, while having no relationship between antibody concentration and inhibition rates of NIH3T3 cells.

\section{DISCUSSION}

At present, there are many expression systems applied to express antibodies and antibody fragments, including the prokaryotic expression system (yeast,
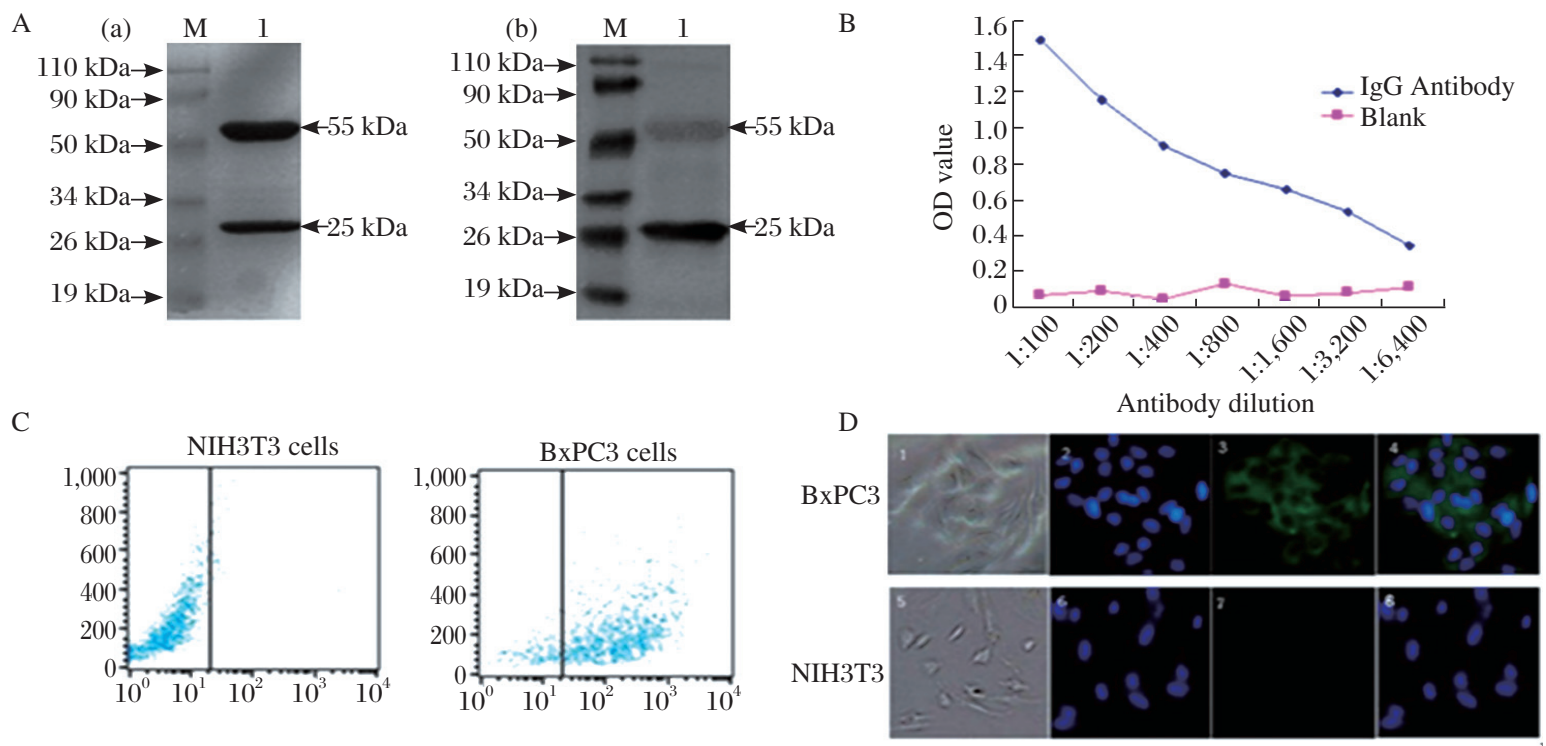

Fig.2 Identification and the immunological function of human anti-TROP2 antibody IgG. A: SDS-PAGE analysis and Western blot analysis of purified antibody. (a) purified antibodies were detected by SDS-PAGE; (b) purified antibodies were detected by Western blot; M: marker, 1: antibody electrophoresis band. B: Analysis of antibody titer of human anti-TROP2 IgG antibody. $P<0.05$. C: Fluorescence-activated cell sorter annlysis: the combination of human anti-TROP2 IgG antibody with BxPC3 and NIH3T3 cell lines. $P<0.05$. D: Immunofluorescence assay, the combination of human anti-TROP2 IgG antibody with BxPC3, NIH3T3 cell lines. 
A

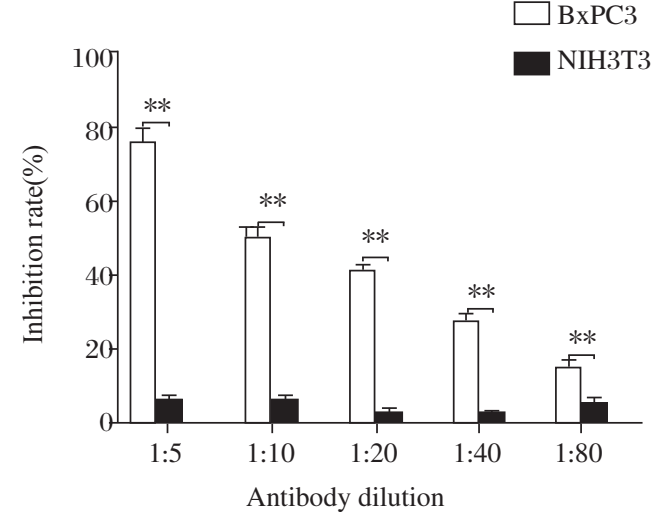

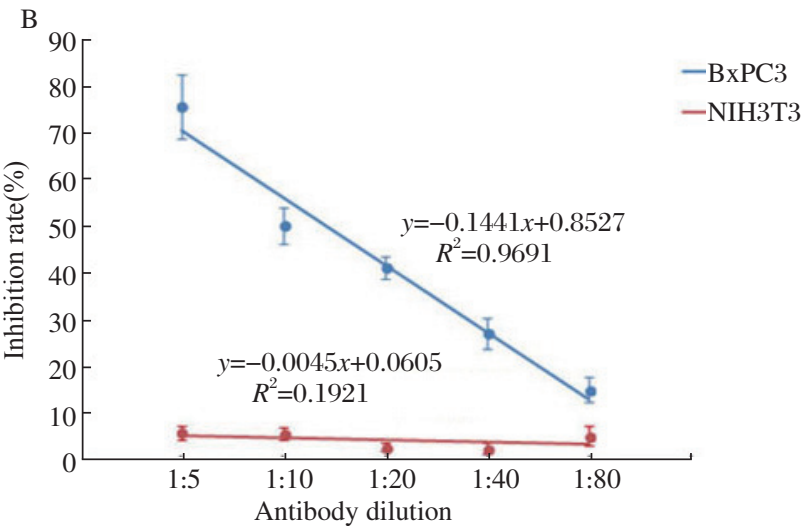

Fig.3 Inhibition effect of human anti-TROP2 antibody IgG on pancreatic cancer cell proliferation. A: Inhibition effect of human anti-TROP2 IgG antibody on proliferation ability of BxPC3 and NIH3T3 cell lines. ${ }^{*} P<0.05$. B: The correlation analysis between the dilutability of antibody and the cell growth inhibition rate. $P<0.05$.

bacteria, etc.) and eukaryotic expression system (mammalian cells, etc.), and each has its advantages and disadvantages. Bacteria are the most mature low cost expression platform, and it is often used in the production of antibody fragments. In contrast, the eukaryotic expression cell has a more complete set of system on synthesis, assembly and secretion of protein components. Compared with other expression systems, there are many advantages in the eukaryotic expression system, for example, the system has many post-translational modification options including the reliable processing of folding antibodies, right connection of glycosylation, amidation, and disulfide bonding, correct assembly of subunits, and so on, thus the generated antibody molecules are able to form interchain disulfide bonds as with natural protein ${ }^{[17-21]}$.

The related studies found that small molecule antibody (such as Fab antibody) has many advantages such as weak immunogenicity, small molecular weight and easy delivery to tumor tissues, however, it also has many shortcomings such as short retention time, easy cleaning, and so on. Therefore, some laboratories devoted themselves to the study of the whole molecular antibody, whose advantages include; (1) fewer mouse derived components, which reduces immunological rejection; (2) high affinity and good stability; (3) the humanized antibody can activate the complement system and the biological function mediated by Fc receptor, which has a lethal effect on target cells; (4) the antibody has long half-life in vivo, the halflife of the humanized antibody can reach up to several days, sometimes even close to $21 \mathrm{~d}$, because the $\mathrm{Fc}$ segment is able to specifically bind to the $\mathrm{Fc}$ receptor $(\mathrm{FcRn})$ on human vascular endothelial cells, so that the antibody can not only resist quick degradation, but can also be released into the blood at any time. Therefore, on the basis of that human anti-TROP2 Fab antibody which had been developed in our laboratory, the authors manufactured and analyzed the human anti-TROP2 antibody IgG.

This experiment successfully constructed the eukaryotic expression system of human anti-TROP2 antibody IgG and obtained pure antibodies. The results showed that the molecule weight of heavy chain and light chain were $55 \mathrm{kDa}$ and $28 \mathrm{kDa}$. The result of ELISA showed human anti-TROP2 antibody IgG could specifically combine with TROP2 antigen in $1: 6,400$ dilution, which indicated that the combination of human anti-TROP2 antibody IgG and TROP2 protein has high specificity and high titer. The result of flow cytometry and immunofluorescence showed that human anti-TROP2 antibody IgG can specifically recognize the TROP2 protein, and the MTT assay results indicated that human anti-TROP2 antibody IgG could inhibit the growth and proliferation of BxPC3 cells obviously. In addition, the results indicated that the inhibition ratio obviously improved with increased antibody concentration. In summary, the results of this study suggest that the human anti-TROP2 antibody IgG eukaryotic expression system be successfully constructed and human anti-TROP2 antibody $\operatorname{IgG}$ be correctly expressed, and the antibody can significantly inhibit the growth and proliferation of pancreatic cancer cells. Therefore, the human anti-TROP2 antibody Ig G has good application prospects in the targeted therapy of pancreatic cancer.

The dihydrofolate reductase (DHFR) system is currently the most commonly used mammalian expression system, which adopted Chinese hamster ovary cell line (CHO cell line) with DHFR defect. These cells cannot synthesize tetrahydrofolate, but DHFR is essential in the biosynthesis of purine, so these cells can grow only on the medium with thymidine, glycine and purine. The vector containing the DHFR gene and 
on pancreatic cancer cell proliferation, 2017, 1(4)

antibody gene were transfected into the $\mathrm{CHO}$ cell line, and then the $\mathrm{CHO}$ cell line was grown in the medium without the above additive. This kind of DHFR function can be inhibited by MTX (belonging to folic acid analogs). The anti MTX cell lines can be selected by continuously improving the concentration of MTX. In this way a large amount of the DHFR gene can be amplified while at the same time, the antibody gene can also be amplified, thus increasing antibody expression. It is generally recognized that, compared with Fab, IgG has a higher affinity, better stability, and is able to activate the complement system and the biological function mediated by the $\mathrm{Fc}$ receptor. Therefore, $\mathrm{CHO}$ $\mathrm{dhfr}^{-}$cells were applied to express human anti-TROP2 antibody $\operatorname{IgG}$, obtaining the whole molecular antibody, which was more similar to the molecular conformation of natural antibodies. On the other hand, the human anti-TROP2 antibody IgG contained $\mathrm{Fc}$ segment, which was able to bind with Protein $G$ in high affinity, and could be purified by the Protein G affinity column, the method being less complex, achieving both heightened efficiency and purity.

\section{Acknowledgments and funding}

This work was supported in part by National Natural Science Foundation of China (No. 81101704) and Nanjing Municipality Medical Technology Development Project (No. ZKX12025).

\section{References}

[1] Lau WY, Lai EC. Development and controversies of adjuvant therapy for pancreatic cancer. Hepatobiliary Pancreat Dis Int,2008,7(2):121-5.

[2] Jiang YJ, Liu MD, Li ZS, et al. Discovery of novel candidate oncogenes in pancreatic carcinoma using high-throughput microarrays. Hepatogastroenterology,2013,60(128):1825-32.

[3] Yee NS, Kazi AA, Yee RK. Translating discovery in zebrafish pancreatic development to human pancreatic cancer: biomarkers, targets, pathogenesis, and therapeutics. Zebrafish,2013,10(2, SI):132-46.

[4] Lin JC, Wu YY, Wu JY, et al. TROP2 is epigenetically inactivated and modulates IGF-1R signalling in lung adenocarcinoma. EMBO Mol Med,2012,4(6):472-85.

[5] Bignotti E, Todeschini P, Calza S, et al. Trop-2 overexpression as an independent marker for poor overall survival in ovarian carcinoma patients. Eur J Cancer,2010,46(5):944-53.

[6] Fong D, Spizzo G, Gostner JM, et al. TROP2: a novel prognostic marker in squamous cell carcinoma of the oral cavity. Mod Pathol,2008,21(2):186-91.

[7] Cubas R, Li M, Chen C, et al. Trop2: a possible therapeutic target for late stage epithelial carcinomas. Biochim Biophys Acta,2009,1796(2):309-14.
[8] Zheng YY. Advances in research and application of domestic monoclonal antibody-targeted therapy for cancer. Chin J Cancer Biother,2006,13(1):70-3.

[9] Wang XY, Lin H, Zhang HL, et al. Preparation and optimization of human anti-Trop-2 antibody fragment Fab. Acta Universitatis Medicinalis Nanjing(Natural Science),2012,32(1):35-9.

[10] Stepan LP, Trueblood ES, Hale K, et al. Expression of Trop2 cell surface glycoprotein in normal and tumor tissues: potential implications as a cancer therapeutic target. J Histochem Cytochem,2011,59(7):701-10.

[11] Liang J, Liu QQ, Zhang HL, et al.Preparation and immunologic characterization of a monoclonal antibody against human trophoblast cell-surface antigen 2 . Acta Universitatis Medicinalis Nanjing (Natural Science),2011,31(5):645-50.

[12] Chen HL, Chen Te, Bi XY. Preparation and identification of monoclonal antibody against NT-proBNP. Chin J Gerontol,2(37):525-8.

[13] Kodama Y, Suzuki H, Dohra H, et al. Comparison of gene expression of Paramecium bursaria with and without Chlorella variabilis symbionts. BMC Genomics,2014,15(1):183.

[14] Nguyen MT, Koo BK, Thi Vu TT, et al.Prokaryotic soluble overexpression and purification of bioactive human growth hormone by fusion to thioredoxin, maltose binding protein, and protein disulfide isomerase. PLoS One,2014,9(3):e89038.

[15] Porowinska D, Wujak M, Roszek K, et al. Prokaryotic expression systems. Postepy Hig Med Dosw (Online), 2013, 67: 119-29.

[16] Barrett LW, Fletcher S, Wilton SD. Regulation of eukaryotic gene expression by the untranslated gene regions and other non-coding elements. Cell Mol Life Sci,2012,69(21):3613-34.

[17] Thanh T, Chi VT, Omar H, et al. Sequence analysis and potentials of the native $\mathrm{RbcS}$ promoter in the development of an alternative eukaryotic expression system using green microalga ankistrodesmus convolutus. Int $J$ Mol Sci,2012,13(3):2676-91.

[18] Xiang JJ, Tong JY, Wang H. Recent advances in antibody technique(1):Humanized antibody technique. Journal of Jinan University(Natural Science),2012,33(5):524-30.

[19] Yu CM, Xu J, Tong YG, et al. Expression of whole human anti_HAV antibody in CHO cells. Bull Acad Mil Med Sci,2002,26(3):176-81.

[20] Wang DY, Cui YP, Jiang ZX, et al. Knockdown expression of eukaryotic initiation factor $5 \mathrm{C}$-terminal domain containing protein extends lifespan in Drosophila melanogaster. Biochem Biophys Res Commun,2014,446(2):465-9.

[21] Wang PL, Bao Y, Yee MC, et al. Circular RNA is expressed across the eukaryotic tree of life. PLoS One,2014,9(3):e90859.

(Received 11 October 2017, 09 November 2017, Accepted 19 November 2017) 